\title{
Risk Factors Affecting Cage Retropulsion into the Spinal Canal Following Posterior Lumbar Interbody Fusion: Association with Diffuse Idiopathic Skeletal Hyperostosis
}

\author{
Shinichi Kato, Nobuki Terada, Osamu Niwa, Mitsuko Yamada \\ Department of Orthopedic Surgery, Restorative Medicine of Neuro-Musculoskeletal System, School of Medicine, Fujita Health University, Nagoya, Japan
}

Study Design: This was a retrospective observational study.

Purpose: We identify risk factors, including physical and surgical factors, and comorbidities affecting cage retropulsion following posterior lumbar interbody fusion (PLIF).

Overview of Literature: Diffuse idiopathic skeletal hyperostosis (DISH) is considered a risk factor for reoperation after PLIF. We evaluated the effect of DISH on cage retropulsion into the spinal canal, which may require surgical revision for severe neurological disorders.

Methods: A total of 400 patients (175 men, 225 women) who underwent PLIF were observed for $>1$ year. Factors investigated included the frequency of cage retropulsion and surgical revision. In addition, physical (age, sex, disease), surgical (fusion and PLIF levels, cage number, grade 2 osteotomy), and comorbid (DISH, existing vertebral fracture) factors were compared between patients with and without cage retropulsion. Factors related to surgical revision during the observation period were also considered.

Results: Cage retropulsion occurred in 15 patients and surgical revision was performed in 11. Revisions included the replacement of pedicle screws (PSs) with larger screws in all patients and supplementary implants in 10. Among the patients with cage retropulsion, the average PLIF level was 2.7, with DISH present in nine patients and existing vertebral fractures in six. Factors affecting cage retropulsion were diagnoses of osteoporotic vertebral fracture, multilevel fusion, single-cage insertion, grade 2 osteotomy, presence of DISH, and existing vertebral fracture. Multivariable analysis indicated that retropulsion of a fusion cage occurred significantly more frequently in patients with DISH and multilevel PLIF.

Conclusions: DISH and multilevel PLIF were significant risk factors affecting cage retropulsion. Revision surgery for cage retropulsion revealed PS loosening, suggesting that implant replacement was necessary to prevent repeat cage retropulsion after revision.

Keywords: Diffuse idiopathic skeletal hyperostosis; Posterior lumbar interbody fusion; Cage retropulsion; Reoperation; Spinal canal

\section{Introduction}

Posterior lumbar interbody fusion (PLIF) has been per- formed for various clinical conditions, because widespread spinal canal decompression and intervertebral stability can be obtained simultaneously while restoring foraminal

Received Aug 24, 2020; Revised Sep 26, 2020; Accepted Sep 27, 2020

Corresponding author: Shinichi Kato

Department of Orthopedic Surgery, Restorative medicine of Neuro-Musculoskeletal System, School of Medicine, Fujita Health University, 3-6-10 Otobashi, Nakagawa-ku, Nagoya, Aichi 454-8509, Japan

Tel: +81-52-323-5650, Fax: +81-52-332-8634, E-mail: shkato@fujita-hu.ac.jp 
height [1]. Recently, PLIF has been applied in pathological conditions to correct spinal deformities or during anterior column reconstruction for vertebral fractures [2,3]. As minimally invasive techniques advance, their indications have expanded to include multiple intervertebral lesions and surgery in elderly patients. Although many surgical outcomes have been reported to date, complications vary depending on when they occur postoperatively, and some patients occasionally require surgical revision. Specifically, these complications include instability due to interbody fusion loss and adjacent segment disease [4]. Cage retropulsion into the spinal canal causing neurological disorders is a serious implant-related complication.

Among patients with comorbidities, those with Parkinson's disease suffer complications frequently during spinal surgery [5]. Also, patients with osteoporosis are more likely to undergo an implant-related reoperation [6]. Recently, diffuse idiopathic skeletal hyperostosis (DISH) has attracted attention, because it can reduce intervertebral flexibility and affect perioperative complications [2]. We examined the factors affecting cage retropulsion following PLIF, including physical and surgery-related factors and comorbidities.

\section{Materials and Methods}

\section{Patient population}

A retrospective observational study was conducted in compliance with the tenets of the Declaration of Helsinki. The study was reviewed and approved by the Institutional Review Board for Clinical Research Ethics at Fujita Health University (IRB approval no., HM 19-123) and the requirement for informed consent from some individual participants was omitted because of the retrospective design of this study .

\section{Inclusion and exclusion criteria}

The inclusion criterion was patients with cage retropulsion into the spinal canal over the posterior margin of the vertebral body. DISH was defined as $>4$ continuous ossification vertebrae per the criteria of Resnick et al. [7]. Grade 2 osteotomy with PLIF had been performed on applicable patients with segmental kyphosis or sagittal malalignment. The indications for surgical revision owing to cage retropulsion were neurological disorders accompanied by clinical symptoms, such as back or leg pain. The exclusion criterion was grade 3 osteotomy or higher with PLIF.

\section{Surgical technique}

As usual, in these procedures, cranial laminectomy and partial facetectomy were performed first. Second, pedicle screws (PSs) were inserted and the PS-attached device was used to perform disc space distraction to prepare the disc, followed by disc space packing with autogenous local bone. Finally, the cage was inserted and the PS systems were fixed. Grade 2 osteotomy was performed after PS insertion to prevent pedicle fracture. Generally, two cages were inserted in each disc space, except in patients with poor overall condition, in whom only one cage was inserted. The implant material used for the cage was polyether ether ketone (PEEK) in all but nine cases. All cages were of the box or bullet type, and the lordosis angle was $\leq 6^{\circ}$.

\section{Patient demographics and surgical, radiological, and clinical examinations}

All demographics and examination results were compared between the patients with and without cage retropulsion. Demographics, such as age, sex, diagnosis, fusion and PLIF levels, cage number, grade 2 osteotomy, presence of $\mathrm{DISH}$, existing vertebral fracture, and revision surgery, were evaluated. In patients with cage retropulsion, clinical examinations, such as the frequency, period, level, Visual Analog Scale (VAS), surgical examinations for revision, and radiological examinations for osseous union at the cage retropulsion level, were evaluated.

Interbody fusion at the cage retropulsion level was assessed by reconstruction computed tomography (CT) at 1 year postoperatively. Sagittal and coronal CT was used to evaluate the fusion condition for the existence of consecutive bone formation in multiple slices obtained around the cage.

\section{Statistical analysis}

All values are expressed as the mean \pm standard deviation. Statistical analyses were performed with respect to the presence of cage retropulsion. Continuous variables (age, fusion and PLIF levels, and VAS) were assessed using the Mann-Whitney $U$-test, and categorical variables (sex, cage number, DISH presence, grade 2 osteotomy, existing ver- 
tebral fracture, and revision surgery) were assessed using Fisher's exact test. The variable (diagnosis) was assessed with the $\chi^{2}$ test. A $p<0.05$ was considered statistically significant. Variables with statistically significant $p$-values on univariate analysis were analyzed by multivariable analysis. Statistical analyses were performed with the JMP ver. 14.2.0 software suite (SAS Institute Inc., Cary, NC, USA).

\section{Results}

\section{Patient demographics, and surgical and clinical out- comes of all patients}

The average age of the 175 men and 225 women was 67.7 years. Diagnoses included lumbar canal stenosis (LCS) in 142 patients, degenerative spondylolisthesis (DS) in 116, lumbar disc hernia in 52, degenerative lumbar scoliosis (DLS) in 41, isthmic spondylolisthesis in 20, degenerative kyphosis in 17, and osteoporotic vertebral fracture (OVF) in 12. The average fusion level was 2.1 and the average PLIF level was 1.8. A total of 30 patients received one cage and 370 received two cages. Grade 2 osteotomy was performed in 76 patients, with DISH present in 98, and existing vertebral fracture in 36 . Surgical revision was performed in 52 patients, including 22 with adjacent segment disease and 11 with cage retropulsion (Table 1).

\section{Patient demographics, and surgical, radiological, and clinical outcomes of patients with cage retropulsion}

The frequency of cage retropulsion was 3.8\% (15/400 patients), and it occurred at 15 of 707 (2.1\%) intervertebral levels. The average age of the seven men and eight women was 72.7 years. Five patients had LCS, five had DS, three had OVF, and two had DLS. The average fusion level was 2.9, the average PLIF level was 2.7, and the average retropulsion period was 16.1 days. Retropulsion levels were $\mathrm{L} 4 / 5$ in six patients, $\mathrm{L} 5 / \mathrm{S}$ in five, $\mathrm{L} 2 / 3$ in three, and $\mathrm{L} 3 / 4$ in one. The VAS at 1 year postoperatively was 3.2 and $3.6 \mathrm{~cm}$ in the groups with and without revision surgery, respectively (the results were not significantly different) (Tables 2,3).

\section{Revision surgery}

Eleven patients underwent surgical revision for cage retropulsion. Laboratory values, including the white blood cell count, C-reactive protein, and neutrophil levels were
Table 1. Patient demographic data

\begin{tabular}{|c|c|}
\hline Characteristic & Value \\
\hline Age (yr) & $67.7 \pm 12.2$ \\
\hline \multicolumn{2}{|l|}{ Sex } \\
\hline Men & 175 \\
\hline Women & 225 \\
\hline \multicolumn{2}{|l|}{ Diagnosis } \\
\hline Lumbar canal stenosis & 142 \\
\hline Degenerative spondylolisthesis & 116 \\
\hline Lumbar disc hernia & 52 \\
\hline Degenerative lumbar scoliosis & 41 \\
\hline Isthmic spondylolisthesis & 20 \\
\hline Degenerative kyphosis & 17 \\
\hline Osteoporotic vertebral fracture & 12 \\
\hline Fusion level & $2.1 \pm 1.3$ \\
\hline 1 & 167 \\
\hline 2 & 132 \\
\hline 3 & 62 \\
\hline 4 & 19 \\
\hline 5 & 8 \\
\hline$\geq 6$ & 12 \\
\hline Posterior lumbar interbody fusion level & $1.8 \pm 0.9$ \\
\hline 1 & 187 \\
\hline 2 & 137 \\
\hline 3 & 60 \\
\hline 4 & 14 \\
\hline 5 & 2 \\
\hline \multicolumn{2}{|l|}{ No. of cages } \\
\hline 1 & 30 \\
\hline 2 & 370 \\
\hline Grade 2 osteotomy & 76 \\
\hline Diffuse idiopathic skeletal hyperostosis presence & 98 \\
\hline \multicolumn{2}{|l|}{ Existing vertebral fracture } \\
\hline Incident VF & TL 0, ML 8 \\
\hline Prevalent VF & TL 19, ML 12 \\
\hline Duplicate VF cases included & 36 \\
\hline \multicolumn{2}{|l|}{ Revision surgery } \\
\hline Adjacent segment disease & 22 \\
\hline Cage retropulsion & 11 \\
\hline Infection & 7 \\
\hline Implant failure & 3 \\
\hline Hematoma & 2 \\
\hline Additional vertebral fracture & 2 \\
\hline Other & 5 \\
\hline
\end{tabular}

Values are presented as mean \pm standard deviation or number. $\mathrm{VF}$, vertebral fracture; TL, thoraco-lumbar; $\mathrm{ML}$, middle-lower lumbar. 
Table 2. Characteristics of cage retropulsion patients

\begin{tabular}{|c|c|c|c|c|c|c|c|}
\hline Age (yr) & Sex & Fusion level & PLIF level & Cage type & Revision surgery & VAS at 1 year postoperatively $(\mathrm{cm})$ & Retropulsion level/fusion \\
\hline 58 & Man & L2-S & 4 & PEEK & + & 3.5 & $\mathrm{~L} 5 / \mathrm{S} /+$ \\
\hline 70 & Man & $\lfloor 4 / 5$ & 1 & PEEK & - & 4.1 & L4/5/- \\
\hline 73 & Man & L2-5 & 3 & PEEK & + & 3.7 & $\mathrm{~L} 2 / 3 /-$ \\
\hline 75 & Man & L3-S & 3 & PEEK & + & 2.5 & L5/S/+ \\
\hline 75 & Man & L2-iliac & 4 & PEEK & + & 2.8 & $\mathrm{~L} 2 / 3 /+$ \\
\hline 81 & Man & L4-S & 2 & PEEK & + & 2.0 & $L 4 / 5 /+$ \\
\hline 86 & Man & L4-S & 2 & PEEK & + & 3.8 & L5/S/+ \\
\hline 49 & Woman & L2-S & 3 & PEEK & + & 4.7 & L5/S/+ \\
\hline 53 & Woman & L4-S & 2 & PEEK & - & 3.1 & L5/S/- \\
\hline 71 & Woman & L2-5 & 2 & PEEK & + & 2.3 & L4/5/+ \\
\hline 72 & Woman & L1-4 & 3 & PEEK & + & 2.6 & L3/4/+ \\
\hline 78 & Woman & L3-5 & 2 & PEEK & - & 3.3 & L4/5/+ \\
\hline 79 & Woman & L2-iliac & 4 & PEEK & + & 4.3 & L2/3/+ \\
\hline 81 & Woman & L2-5 & 3 & PEEK & + & 3.3 & L4/5/+ \\
\hline 89 & Woman & L2-5 & 3 & PEEK & - & 3.7 & L4/5/- \\
\hline
\end{tabular}

PLIF, posterior lumbar interbody fusion; VAS, Visual Analog Scale; PEEK, polyether ether ketone.

Table 3. Univariable and multivariable analysis of cage retropulsion patients versus non-cage retropulsion patients

\begin{tabular}{|c|c|c|c|c|}
\hline Variable & Cage retropulsion & Non-cage retropulsion & Univariable $p$-value & Multivariable $p$-value \\
\hline Age (yr) & 72.7 & 67.6 & 0.11 & - \\
\hline Sex & & & 0.81 & - \\
\hline Men & 7 & 168 & & \\
\hline Women & 8 & 217 & & \\
\hline Diagnosis & & & $0.03^{\mathrm{al}}$ & 0.3436 \\
\hline Lumbar canal stenosis & 5 & 137 & & \\
\hline Degenerative spondylolisthesis & 5 & 111 & & \\
\hline Osteoporotic vertebral fracture & 3 & - & & \\
\hline Lumbar disc hernia & - & 52 & & \\
\hline Degenerative lumbar scoliosis & 2 & 39 & & \\
\hline Isthmic spondylolisthesis & - & 20 & & \\
\hline Degenerative kyphosis & - & 17 & & \\
\hline Osteoporotic vertebral fracture & - & 9 & & \\
\hline Fusion level & 2.9 & 2.0 & $0.0098^{\mathrm{a})}$ & 0.2994 \\
\hline Posterior lumbar interbody fusion level & 2.7 & 1.7 & $<0.0001^{\text {a) }}$ & $0.0157^{\mathrm{a})}$ \\
\hline No. of cages & & & $0.0206^{\mathrm{al}}$ & 0.9707 \\
\hline 1 & 4 & 26 & & \\
\hline 2 & 11 & 359 & & \\
\hline Grade 2 osteotomy & 6 & 70 & $0.0388^{\mathrm{a})}$ & 0.2378 \\
\hline Diffuse idiopathic skeletal hyperostosis presence & 9 & 89 & $0.0011^{\mathrm{a})}$ & $0.0165^{a)}$ \\
\hline Existing vertebral fracture & 6 & 30 & $<0.0001^{\text {a) }}$ & 0.1377 \\
\hline Revision surgery & 11 & 41 & $<0.0001^{\text {al }}$ & $<0.0001^{\text {a) }}$ \\
\hline
\end{tabular}

${ }^{a}$ Statistically significant. 


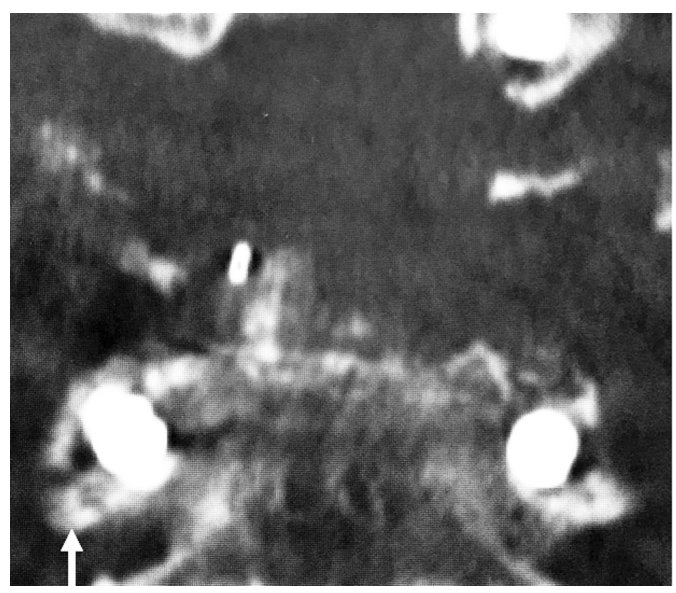

Fig. 1. Cage retropulsion accompanied by pedicle fracture (arrow) on coronal computed tomography.

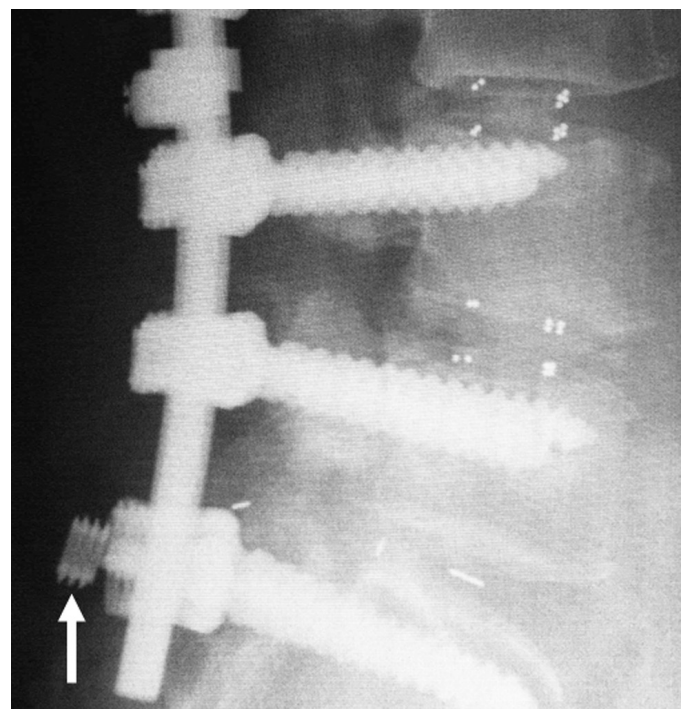

Fig. 2. Cage retropulsion accompanied with set screw dislocation (arrow) on lateral radiograph.

lower at revision than after the initial surgery. The findings at revision included PS loosening in all patients and pedicle fractures in three not present at the initial operation (Fig. 1), and PS set screw dislocation in one (Fig. 2). Clear pus effusion indicative of infection was not found intraoperatively in any patient. Revisions included the replacement of PSs with a larger screw and the insertion of the same cage as previously in all patients. Supplementary implants were inserted in 10 patients, including the addition of posterolateral lumbar fusion in five, iliac screws in four, and PLIF in one (Fig. 3). The average operative time was 111 minutes (range, 64-191 minutes), and the average blood volume loss was $245 \mathrm{~mL}$ (range, 50-730 mL). Perioperative complications, including neurological disorders

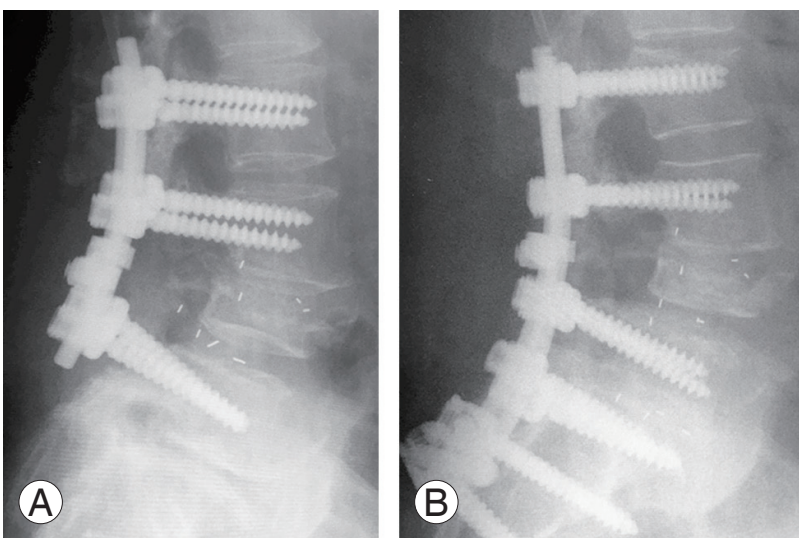

Fig. 3. (A) Cage retropulsion occurred at the $L 4 / 5$ level on lateral radiograph. (B) Revision surgery performed for posterior lumbar interbody fusion between $L 5$ and $S$ with an additional iliac screw on lateral radiograph.

and repeat cage retropulsion and infection, did not occur in any patient. Intervertebral bone fusion at the cage retropulsion level was noted in 10 patients.

\section{Patient demographics, and surgical and radiological outcomes of patients with and without cage retropul- sion}

On univariate analysis, the average age was 72.7 and 67.6 years in patients with and without cage retropulsion, respectively (not significantly different). There was no significant difference between sexes. The diagnoses of patients with and without cage retropulsion, and with LCS, DS, OVF, and DLS, were significantly different $(p=0.03)$, as were the average fusion $(p=0.0098)$ and PLIF $(p<0.0001)$ levels with and without cage retropulsion. Significant differences were seen among the groups with one and two cages placed ( $p=0.0206$ ), and among those who underwent grade 2 osteotomy ( $p=0.0388$ ). The presence of DISH in the two groups and of existing vertebral fractures were also significantly different $(p=0.0011$ and $p<0.0001$, respectively). Revision was performed in 11 patients with cage retropulsion and in 41 without cage retropulsion (significantly different, $p<0.0001$ ).

On multivariable analysis, the PLIF level $(p=0.0157)$, DISH presence $(p=0.0165)$, and revision surgery $(p<0.0001)$ were significantly different (Table 3 ).

\section{Discussion}

We identified risk factors affecting cage retropulsion following PLIF. Initially, in the PLIF procedure, an iliac bone 


\begin{tabular}{|c|c|c|c|c|c|}
\hline Reference & $\begin{array}{l}\text { Age } \\
\text { (yr) }\end{array}$ & $\begin{array}{c}\text { Frequency } \\
(\%)\end{array}$ & Diagnosis & $\begin{array}{l}\text { Revision } \\
\text { surgery (\%) }\end{array}$ & Risk factor \\
\hline Aoki et al. [11] (2010) & NA & $4 / 125(2.8)$ & DS 4 & $2 / 4(50)$ & Cage type; disc height \\
\hline Kimura et al. [12] (2012) & 68.2 & $9 / 1,070(0.8)$ & LCS 6; DLS 3 & $3 / 9(33)$ & Pear-shaped disk; disc height; disc ROM; multilevel fusions (L5/S) \\
\hline Pan et al. [13] (2016) & 45.6 & $8 / 667(1.2)$ & LCS 3; LDH 3; DLS 1 & $6 / 8(75)$ & Infection \\
\hline Li et al. [14] (2017) & 45.4 & $18 / 286(6.3)$ & DLS 7; LDH 6; LCS 5 & NA & Surgeons experience; cage type; spondylolisthesis \\
\hline Lee et al. [15] (2018) & 68.3 & $16 / 1,047(1.5)$ & LCS 9; DLS 5 & $0 / 16(0)$ & Low BMI; pear-shaped disc; pedicle screw loosening \\
\hline This study & 72.7 & $15 / 400(3.8)$ & LCS 5; DS 5; OVF 3; DLS 2 & $11 / 15(73)$ & DISH; multilevel PLIF \\
\hline
\end{tabular}

NA, not available; DS, degenerative spondylolisthesis; LCS, lumbar canal stenosis; DLS, degenerative lumbar scoliosis; ROM, range of motion; LDH, lumbar disc hernia; $\mathrm{BMI}$, body mass index; OVF, osteoporotic vertebral fracture; DISH, diffuse idiopathic skeletal hyperostosis; PLIF, posterior lumbar interbody fusion.

autograft was transplanted only into the interbody space after posterior decompression. Therefore, pseudoarthrosis accompanied by retropulsion or collapse of the grafted bone was of great concern [4], and the combined use of cages [8] and PSs [1] was added to PLIF.

PLIF performed without PS results in a significantly greater incidence of cage retropulsion [9], and sometimes reoperation with additional PS fixation is required. Nevertheless, once interbody fusion is determined and the PS is removed, cage retropulsion can occur [10]. Cage retropulsion into the spinal canal can cause not only neurological disorders but also decreased lumbar lordosis, causing spinal canal or foraminal stenosis and interbody pseudarthrosis due to loss of correction. Therefore, recently, the use of PSs has become common in cases of PLIF.

Limited reports exist of cage retropulsion after lumbar interbody fusion combined with PS fixation [11-15] (Table 4). Cage retropulsion was related to the physical factors of the L5/S level and intervertebral height [12]. In five of our six patients with cage retropulsion, in which the L5/S level was included in the caudal margin, cage retropulsion occurred at the L5/S level. In addition, in each patient with $\geq 3$ fusion levels without iliac screw fixation, cage retropulsion occurred at the fixed end, particularly on the caudal side (seven of eight patients). Our results indicated that more caution is required when the L5/S level is at the caudal margin of the multilevel interbody fusion owing to cage retropulsion.

Factors reportedly related to surgery include multilevel fusion [12], the number of cages [16], and total facetectomy [9], which are the same factors found in our study. Among the cases reported by Kimura et al. [12], the patients' average age was 68.2 years and the average number of PLIF levels was 1.7. Of these cases, single-level PLIF accounted for more than half of those with cage retropulsion. The frequency was $0.8 \%$, and approximately $70 \%$ of the diagnoses were of LCS. Compared to our study, their frequency rate was lower, but the average age, number of PLIF levels, and targeted diseases were different. In our study, cage retropulsion tended to occur after PLIF for OVFs. Our results are thought to be meaningful when evaluating complications in relatively older patients undergoing multilevel PLIF.

Few studies exist on the association between comorbidities and cage retropulsion [14]. In our patients, DISH was a risk factor for cage retropulsion following PLIF. DISH is considered a risk factor for reoperation after interbody fusion [17]. However, to date, no reports have associated DISH with implant complications, to our knowledge. The association between DISH and cage retropulsion may be because of the concentration of stress from the long lever arm due to continuous ossification, which can influence the intervertebral space. Furthermore, unequal intervertebral pressure exerted by one cage, extensive decompression accompanied by multilevel fusion, or posterior instability due to grade 2 osteotomy might also influence cage retropulsion. Based on a report of the involvement of sagittal movements of the spine in cage retropulsion [18], sagittal malalignment due to vertebral fracture can cause an increase in intervertebral pressure and might contribute to posterior cage retropulsion.

The bone mineral density contributes to pressure between the intervertebral bone graft and endplate [19]. However, the relationship between cage retropulsion and osteoporosis is considered weak $[12,14,15]$. In contrast, PS loosening was noted during all our reoperations for cage retropulsion, and as expected, the interbody pressure was diminished. Moreover, osteoporosis is involved in PS 
loosening, which reduces the interbody fusion rate following PLIF [20].

DISH tends to be associated with osteoporosis when it is accompanied by vertebral fractures [21]. The most frequent cause of reoperation within 1 year was instrument failure [6]. In addition, reported risk factors of PS loosening include advanced age, low bone mineral density, and $\geq 3$ levels of fusion [22]. These results suggested that PSs are likely to loosen in the case of multilevel interbody fusion, especially in elderly people with vertebral fracture and DISH, and this may lead to a high risk of cage retropulsion.

At reoperation for cage retropulsion, an approach from the same incision is considered high risk because of concern for nerve injury caused by tissue adhesion [9]. However, when reoperation is performed via an anterior approach, complications, such as vascular injury, may occur. To date, detailed reports on reoperation for cage retropulsion have been limited [23].

Eleven of our patients underwent reoperation for cage retropulsion. In all approaches, we used the same posterior incision, and the cage could be set up without intraoperative complications, such as nerve injury. We increased the size of the PSs in all patients and, although we set up the same cage as previously, there were no instances of repeat cage retropulsion after reoperation. There is a report that repeat reoperation for recurrent cage retropulsion was necessary to increase the size of the cage or PS when the same size of PS and cage was used at reoperation [24]. As a small cage size can be a risk factor for retropulsion $[11,14]$, changing the size of either implant at reoperation is likely to be required. An additional implant was inserted in all but one of our patients, which might have helped stabilize the cage retropulsion.

Interbody fusion was recognized after cage retropulsion in 10 of the 11 reoperated patients compared to only one of the four in whom reoperation was not performed. Therefore, in cases of cage retropulsion after PLIF, interbody fusion is expected to be difficult through the natural course of healing. On the other hand, regarding clinical outcomes, the VAS was not different between patients with and without reoperation.

Based on the results, reoperation is considered desirable for cases exhibiting favorable overall conditions if the complication of cage retropulsion occurs during the early postoperative period. Reoperation following PLIF was significantly more likely to occur in patients with cage retropulsion and, thus, its prevention is important. Aggressive treatment of osteoporosis may prevent PS loosening [25] and increase the osseous union rate of PLIF [26], which may reduce the risk of cage retropulsion. Performing multilevel PLIF may increase not only the risk of cage retropulsion but also surgical invasiveness. Recently, lateral interbody fusion (LIF) has become popular in surgery for adults with spinal deformities, as it is minimally invasive while resulting in satisfactory lumbar lordosis [27]. Furthermore, because the posterior longitudinal ligament is not sacrificed during the LIF procedure, cage retropulsion into the spinal canal is less likely to occur. Therefore, the combination with LIF appears to be a useful option when performing multilevel interbody fusion.

Our study had some limitations. This was a singlecenter, retrospective study. We did not examine the bone mineral density in patients with cage retropulsion. Therefore, the effect of osteoporosis on PS loosening accompanied by cage retropulsion was uncertain. As the follow-up periods varied widely, the frequency of adjacent segment disease, which is the most frequent cause of reoperation, could not be evaluated accurately. We did not examine tissue membrane cultures or pathology in the tract of a loosened PS or retropulsed cage. Therefore, the possibility of subclinical infection cannot be denied. We limited our selection of cage material primarily to PEEK and size to a lordosis angle $\leq 6^{\circ}$, and these factors may have contributed to cage retropulsion.

\section{Conclusions}

Cage retropulsion following PLIF occurred significantly frequently in the presence of DISH and multilevel fusion, particularly when multilevel PLIF was performed. Surgical revision was more frequent in patients with cage retropulsion and was accompanied by PS loosening, suggesting that the replacement of the implant, particularly the PSs, was necessary to prevent repeated cage retropulsion after revision.

\section{Conflict of Interest}

No potential conflict of interest relevant to this article was reported.

\section{Author Contributions}

Shinichi Kato: conception and design, analysis of data, 
drafting of the manuscript, critical revision; Nobuki Terada: administrative support; Osamu Niwa: data acquisition; and Mitsuko Yamada: administrative support.

\section{References}

1. Steffee AD, Sitkowski DJ. Posterior lumbar interbody fusion and plates. Clin Orthop Relat Res 1988;227:99102.

2. Kato S, Terada N, Niwa O. Surgical treatment of osteoporotic vertebral fracture associated with diffuse idiopathic skeletal hyperostosis along with comparative assessment of the levels of affected vertebra or anterior column reconstruction. Spine Surg Relat Res 2019;4:57-63.

3. Wu CH, Wong CB, Chen LH, Niu CC, Tsai TT, Chen WJ. Instrumented posterior lumbar interbody fusion for patients with degenerative lumbar scoliosis. J Spinal Disord Tech 2008;21:310-5.

4. Wetzel FT, LaRocca $H$. The failed posterior lumbar interbody fusion. Spine (Phila Pa 1976) 1991;16:83945.

5. Bouyer B, Scemama C, Roussouly P, et al. Evolution and complications after surgery for spine deformation in patients with Parkinson's disease. Orthop Traumatol Surg Res 2017;103:517-22.

6. Puvanesarajah V, Shen FH, Cancienne JM, et al. Risk factors for revision surgery following primary adult spinal deformity surgery in patients 65 years and older. J Neurosurg Spine 2016;25:486-93.

7. Resnick D, Shaul SR, Robins JM. Diffuse idiopathic skeletal hyperostosis (DISH): Forestier's disease with extraspinal manifestations. Radiology 1975;115:51324.

8. Brantigan JW, Steffee AD, Geiger JM. A carbon fiber implant to aid interbody lumbar fusion: mechanical testing. Spine (Phila Pa 1976) 1991;16:S277-82.

9. Chen L, Yang H, Tang T. Cage migration in spondylolisthesis treated with posterior lumbar interbody fusion using BAK cages. Spine (Phila Pa 1976) 2005;30:2171-5.

10. Kim MH, Kim SW, Kim SH, Kim HS. Extraforaminal lumbar interbody fusion for cage migration after screw removal: a case report. Korean J Spine 2013;10:104-6.

11. Aoki Y, Yamagata M, Nakajima F, et al. Examining risk factors for posterior migration of fusion cages following transforaminal lumbar interbody fusion: a possible limitation of unilateral pedicle screw fixation. J Neurosurg Spine 2010;13:381-7.

12. Kimura H, Shikata J, Odate S, Soeda T, Yamamura S. Risk factors for cage retropulsion after posterior lumbar interbody fusion: analysis of 1070 cases. Spine (Phila Pa 1976) 2012;37:1164-9.

13. Pan FM, Wang SJ, Yong ZY, Liu XM, Huang YF, Wu DS. Risk factors for cage retropulsion after lumbar interbody fusion surgery: series of cases and literature review. Int J Surg 2016;30:56-62.

14. Li $H$, Wang $H$, Zhu $Y$, Ding $W$, Wang $Q$. Incidence and risk factors of posterior cage migration following decompression and instrumented fusion for degenerative lumbar disorders. Medicine (Baltimore) 2017;96:e7804.

15. Lee DY, Park YJ, Song SY, Jeong ST, Kim DH. Risk factors for posterior cage migration after lumbar interbody fusion surgery. Asian Spine J 2018;12:59-68.

16. Aoki Y, Yamagata M, Ikeda Y, et al. A prospective randomized controlled study comparing transforaminal lumbar interbody fusion techniques for degenerative spondylolisthesis: unilateral pedicle screw and 1 cage versus bilateral pedicle screws and 2 cages. J Neurosurg Spine 2012;17:153-9.

17. Otsuki B, Fujibayashi S, Takemoto M, Kimura H, Shimizu T, Matsuda S. Diffuse idiopathic skeletal hyperostosis (DISH) is a risk factor for further surgery in short-segment lumbar interbody fusion. Eur Spine J 2015;24:2514-9.

18. Uzi EA, Dabby D, Tolessa E, Finkelstein JA. Early retropulsion of titanium-threaded cages after posterior lumbar interbody fusion: a report of two cases. Spine (Phila Pa 1976) 2001;26:1073-5.

19. Lim TH, Kwon $\mathrm{H}$, Jeon $\mathrm{CH}$, et al. Effect of endplate conditions and bone mineral density on the compressive strength of the graft-endplate interface in anterior cervical spine fusion. Spine (Phila Pa 1976) 2001;26:951-6.

20. Cho JH, Hwang CJ, Kim H, Joo YS, Lee DH, Lee CS. Effect of osteoporosis on the clinical and radiological outcomes following one-level posterior lumbar interbody fusion. J Orthop Sci 2018;23:870-7.

21. Diederichs G, Engelken F, Marshall LM, et al. Diffuse idiopathic skeletal hyperostosis (DISH): relation to vertebral fractures and bone density. Osteoporos Int 2011;22:1789-97. 
22. Kim JB, Park SW, Lee YS, Nam TK, Park YS, Kim YB. The effects of spinopelvic parameters and paraspinal muscle degeneration on s1 screw loosening. J Korean Neurosurg Soc 2015;58:357-62.

23. Nguyen HV, Akbarnia BA, van Dam BE, et al. Anterior exposure of the spine for removal of lumbar interbody devices and implants. Spine (Phila Pa 1976) 2006;31:2449-53.

24. Lee JG, Lee SM, Kim SW, Shin H. Repeated migration of a fusion cage after posterior lumbar interbody fusion. Korean J Spine 2013;10:25-7.

25. Ohtori S, Inoue G, Orita S, et al. Comparison of teriparatide and bisphosphonate treatment to reduce pedicle screw loosening after lumbar spinal fusion surgery in postmenopausal women with osteoporosis from a bone quality perspective. Spine (Phila $\mathrm{Pa}$ 1976) 2013;38:E487-92.

26. Ebata S, Takahashi J, Hasegawa T, et al. Role of weekly teriparatide administration in osseous union enhancement within six months after posterior or transforaminal lumbar interbody fusion for osteoporosis-associated lumbar degenerative disorders: a multicenter, prospective randomized study. J Bone Joint Surg Am 2017;99:365-72.

27. Watkins RG 4th, Hanna R, Chang D, Watkins RG 3rd. Sagittal alignment after lumbar interbody fusion: comparing anterior, lateral, and transforaminal approaches. J Spinal Disord Tech 2014;27:253-6. 